\title{
Validação Clínica da Versão Portuguesa do Questionário de Hábitos de Sono das Crianças (CSHQ-PT) em Crianças com Perturbações do Sono e PHDA
}

\section{Clinical Validation of The Portuguese Version of the Children Sleep Habits Questionnaire (CSHQ-PT) in Children with Sleep Disorder and ADHD}

Ana Filipa PARREIRA ${ }^{1}$, António MARTINS², Filipa RIBEIRO ${ }^{3}$, Filipe Glória SILVA $\rrbracket^{1}$

Acta Med Port 2019 Mar;32(3):195-201 - https://doi.org/10.20344/amp.10906

\section{RESUMO}

Introdução: A versão Portuguesa do Children's Sleep Habits Questionnaire mostrou propriedades psicométricas adequadas numa amostra comunitária mas o ponto de corte americano pareceu desadequado. O objetivo deste estudo foi validar este questionário em populações clínicas de crianças com distúrbios do sono e com perturbação de hiperatividade/ défice de atenção.

Material e Métodos: Participaram no estudo 148 crianças Portuguesas com idades entre os 2 e os 10 anos, divididas em três grupos: 1. Grupo clínico com perturbação do sono (insónia comportamental, parassónias ou perturbação respiratória do sono); 2 . Grupo clínico com perturbação de hiperatividade/ défice de atenção; 3 . Grupo controlo. Os hábitos e problemas do sono foram avaliados através do Children's Sleep Habits Questionnaire. A perturbação respiratória do sono foi confirmada por polissonografia.

Resultados: A coerência interna do questionário ( $\alpha$ de Cronbach) na amostra clínica (perturbações do sono e perturbação de hiperatividade/ défice de atenção) foi de 0,75 e variou de 0,55 a 0,85 nas subescalas. As crianças com perturbações do sono e com perturbação de hiperatividade/ défice de atenção apresentaram um índice de perturbação do sono mais elevado do que o grupo controlo. As subescalas apresentaram diferenças significativas nos subgrupos de perturbações do sono revelando validade discriminante. A análise receiver operating characteristic do índice de perturbação do sono do grupo com perturbação do sono versus grupo controlo determinou um ponto de corte de 48.

Discussão: As crianças com perturbações do sono e com perturbação de hiperatividade/ défice de atenção apresentaram um Índice de Perturbação do Sono mais elevado do que o grupo controlo. As subescalas apresentaram diferenças significativas nos subgrupos de perturbações do sono revelando validade discriminante.

Conclusão: A versão Portuguesa do Children's Sleep Habits Questionnaire mostrou propriedades psicométricas adequadas em crianças com perturbações do sono e/ou perturbação de hiperatividade/ défice de atenção. O ponto de corte de 48 é mais adequado para a população Portuguesa.

Palavras-chave: Criança; Distúrbios da Iniciação e Manutenção do Sono; Inquéritos e Questionários Sono; Perturbação de Hiperatividade e Défice de Atenção; Portugal

\section{ABSTRACT}

Introduction: The Portuguese version of the Children's Sleep Habits Questionnaire showed adequate psychometric properties in a community sample but the American cut-off seemed inadequate. This study aimed to validate this questionnaire in clinical populations of children with sleep disorders and with attention deficit/ hyperactivity disorder.

Material and Methods: The study sample included 148 Portuguese children aged 2 to 10 years old that where divided in 3 groups: 1. Clinical group with sleep disorders (behavioral insomnias, parasomnias or sleep-related breathing disorders); 2. Clinical group with attention deficit/ hyperactivity disorder; 3. Control group. The sleep habits and sleep problems were evaluated using the Children's Sleep Habits Questionnaire. Sleep-related disorders were confirmed by polysomnography.

Results: The questionnaire's internal consistency (Cronbach $\alpha$ ) in the clinical sample (sleep disorders and attention deficit/ hyperactivity disorder) was 0.75 and ranged from 0.55 to 0.85 for the subscales. Children with sleep disorders and attention deficit/ hyperactivity disorder had a higher sleep disturbance index (full scale score) compared to the control group. The subscales presented significant differences between the subgroups with different sleep disorders showing discriminative validity. The receiver operating characteristic analysis of the sleep disturbance index comparing the sleep disorder and control sample determined a cut-off of 48 (sensibility 0.83 specificity 0.69).

Discussion: Children with sleep disorders and attention deficit/ hyperactivity disorder evidenced higher Sleep Disturbance Index (full scale score) comparing to the control group. The subscales presented significative differences between the subgroups with different sleep disorders showing discriminative validity

Conclusion: The Portuguese version of the Children's Sleep Habits Questionnaire showed adequate psychometric properties for children with sleep disorders and/or attention deficit/ hyperactivity disorder. The cut-off value 48 is better adjusted for the Portuguese population.

Keywords: Attention Deficit Disorder with Hyperactivity; Child; Portugal; Sleep; Sleep Initiation and Maintenance Disorders; Surveys and Questionnaires

\footnotetext{
1. Unidade de Neurodesenvolvimento. Centro da Criança e do Adolescente. CUF Descobertas Hospital. Lisboa. Portugal.

2. Laboratório de Neurofisiologia. CUF Descobertas Hospital. Lisboa. Portugal.

3. Centro de Investigação Interdisciplinar em Saúde. Universidade Católica Portuguesa. Lisboa. Portugal.

$\square$ Autor correspondente: Filipe Glória Silva. fs.sono@gmail.com

Recebido: 10 de julho de 2018 - Aceite: 02 de novembro de 2018 | Copyright @ Ordem dos Médicos 2019
} 


\section{INTRODUÇÃO}

O sono constitui um processo básico dos seres humanos, sendo que passam cerca de um terço da vida a dormir. ${ }^{1}$ As perturbações ou distúrbios do sono atingem cerca de $25 \%$ das crianças em alguma altura da sua vida e podem estar subdiagnosticados. ${ }^{2-4}$ Algumas populações, como as crianças com perturbação de hiperatividade e défice de atenção (PHDA), evidenciam maior prevalência de perturbações do sono do que a população em geral. ${ }^{5}$ Estas perturbações podem ter impacto no desempenho cognitivo e académico das crianças, na regulação emocional e no comportamento, no risco de quedas acidentais e de obesidade. ${ }^{4,6}$ Deste modo, é bastante importante rastrear as perturbações de sono para intervir de forma precoce, resultando assim crianças com maior capacidade de aprendizagem, mais saudáveis e mais felizes. ${ }^{7}$

O Questionário de Hábitos de Sono das Crianças (Children's Sleep Habits Questionnaire) foi desenvolvido nos Estados Unidos com o intuito de avaliar os hábitos e perturbações do sono das crianças, sendo um dos questionários de sono mais utilizados a nível internacional. ${ }^{3,6,7} \mathrm{~A}$ versão Portuguesa (CSHQ-PT) foi validada numa amostra comunitária de crianças dos 2 aos 10 anos mostrando propriedades psicométricas adequadas. Contudo, o ponto de corte sugerido para o questionário original (41) não pareceu adequado para a população Portuguesa por envolver padrões comportamentais influenciados pela cultura, que correspondem a cotações médias superiores na população Portuguesa. ${ }^{3,4}$

Este estudo teve como objetivo validar o CSHQ-PT na população clínica, em crianças com diagnóstico de perturbação do sono e crianças com PHDA, conhecidas por apresentarem maior prevalência deste tipo de problemas.

\section{MATERIAL E MÉTODOS}

A amostra do estudo incluiu crianças de 2 a 10 anos recrutadas numa unidade de saúde privada da região de Lisboa com acordo com múltiplos seguros e subsistemas de saúde. A amostra clínica de crianças com perturbações do sono (grupo-sono) foi recrutada em dois contextos: a) Consulta do Sono, com avaliação clínica realizada por médico com diferenciação nesta área e diagnóstico de perturbação do sono de acordo com os critérios da International Classification of Sleep Disorders, $3^{\text {a }}$ edição (ICSD-3), ${ }^{8}$ divididas em subgrupos de insónia comportamental, parassónias e perturbação respiratória obstrutiva do sono; b) Laboratório de Neurofisiologia, crianças referenciadas para realizar uma polissonografia com índice de apneia-hipopneia (IAH) igual ou superior a um evento por hora, à semelhança do estudo original de validação de Owens et al. ${ }^{3}$ Os parâmetros utilizados para a identificação de distúrbio respiratório nas crianças foram as seguintes: um evento de apneia correspondendo a uma redução em pelo menos $90 \%$ da amplitude medida no termísor nasal; a hipopneia correspondendo a uma redução de pelo menos $30 \%$ da amplitude do sinal na cânula de pressão nasal e acompanhada de dessaturação igual ou superior a $3 \%$ ou seguida de arousal; duração dos eventos de, pelo menos, dois ciclos respiratórios; a pletismografia respiratória de indutância (RIP) para classificação dos eventos. A polissonografia foi realizada nas situações de suspeita de perturbação respiratória do sono na sequência da avaliação clínica inicial. As crianças com parassónias e IAH igual ou superior a 1 foram incluídas no subgrupo clínico de perturbação respiratória obstrutiva do sono. As restantes crianças com diagnóstico de parassónias foram incluídas no subgrupo clínico das parassónias, podendo apresentar insónia comportamental como comorbilidade.

Uma segunda amostra clínica de crianças, com diagnóstico de PHDA de acordo com os critérios do DSM-5 (grupo-PHDA), ${ }^{9}$ foi recrutada na consulta de Pediatria do Desenvolvimento, incluindo crianças com diagnóstico recente (na segunda ou terceira consulta) que não tivessem ainda intervenção comportamental ou farmacológica que pudesse influenciar o padrão de sono, bem como evidência de roncopatia que pudesse configurar perturbação respiratória do sono. A forma de apresentação da PHDA foi especificada (DSM-5). ${ }^{9}$

O grupo-controlo foi recrutado na consulta de vigilância pediátrica (observação de rotina de crianças saudáveis), sendo excluídas as crianças que apresentassem um problema do sono na avaliação dos pais ou diagnóstico prévio de alguma condição que pudesse influenciar o padrão de sono.

O sono foi avaliado através do Questionário de Hábitos do Sono das Crianças (CSHQ-PT), um questionário de 33 itens, previamente validado, que foi preenchido pelos pais. ${ }^{3,10}$ Este instrumento avalia os comportamentos relacionados com o sono que ocorreram na última semana ou numa semana recente típica. As respostas são dadas numa escala de resposta de três pontos: 'Habitualmente', se ocorre cinco a sete vezes por semana; 'Às vezes' se ocorre duas a quatro vezes por semana; 'Raramente', se ocorre uma vez por semana ou se nunca ocorreu. A cada resposta é atribuída uma pontuação que é mais elevada quando corresponde a um comportamento mais problemático. O conjunto de 33 itens é utilizado para determinar a cotação total ou índice de perturbação do sono (IPS) e a cotação de 8 subescalas de problemas do sono: 'Resistência em ir para a cama', 'Início do sono', 'Duração do sono', 'Ansiedade associada ao sono', 'Despertares noturnos', 'Parassónias', 'Perturbação respiratória do sono' e 'Sonolência diurna'. Assim, uma cotação mais elevada nestas escalas corresponde a mais problemas do sono. Foram também incluídas questões sobre dados demográficos, problemas de saúde e utilização regular de medicamentos. Os problemas de saúde foram inquiridos através da pergunta "Algum médico, psicólogo ou terapeuta da fala diagnosticou algum dos seguintes problemas no seu filho?" com as seguintes opções de resposta: rinite, asma, eczema, epilepsia, perturbação de hiperatividade/ défice de atenção, perturbação do espectro do autismo/ síndroma de Asperger, perturbação da linguagem que necessite de terapia da fala, perturbação 
da leitura/ dislexia, outra/ qual

O protocolo deste estudo foi previamente aprovado por Comissão de Ética e foi obtido consentimento informado dos pais. A análise estatística dos dados foi realizada através do programa Statistical Package for the Social Sciences (SPSS), versão 23. A análise das diferenças nas características demográficas entre o grupo clínico e o grupo controlo, como a pertença a um grupo etário ou o nível educacional dos pais, foi efetuada através de uma análise de frequências com o teste de $\chi^{2}$. As cotações das escalas do CSHQ foram comparadas de acordo com o perfil clínico, através de testes não paramétricos Mann-Whitney para dois grupos amostrais; para a comparação de mais de dois grupos amostrais foi utilizado o teste de Kruskal-Wallis. Foi considerado o nível de significância de 0,05 em todas as análises. Verificou-se também a coerência interna do questionário determinando o alfa de Cronbach. A partir da análise de curvas receiver operating characteristic (ROC), determinou-se a sensibilidade e a especificidade de um ponto de corte (cutoff) da cotação do questionário para o rastreio de perturbação do sono. ${ }^{11}$

\section{RESULTADOS}

Foi distribuído um total de 248 questionários e foram devolvidos 175 (70,9\%). Destes, foram excluídos 27: dez por apresentarem critérios de exclusão, seis por apresentarem polissonografias com IAH $<1 / \mathrm{h}$, oito por excederem o limite de idade e três por apresentarem menos de $80 \%$ de respostas válidas. No final, ficaram 148 questionários para análise.

Os subgrupos amostrais tiveram a seguinte constituição: 85 crianças pertenciam ao grupo clínico-sono (31 crianças com diagnóstico de insónia comportamental, 19 crianças com diagnóstico de parassónias e 35 crianças com diagnóstico de perturbação respiratória obstrutiva do sono), 27 pertenciam ao grupo clínico-PHDA (16 crianças com subtipo combinado, 10 crianças com o subtipo predominantemente desatento, uma criança com predomínio de hiperactividade/impulsividade) e 36 crianças constituíam o grupo controlo. No subgrupo Parassónias, 8 crianças (42\%) apresentam comorbilidade com insónia comportamental.

A idade média da amostra total foi de 5,82 anos (d.p $=2,61$ ). Considerando os grupos amostrais, grupo clínico-sono, grupo clínico-PHDA e grupo controlo, verificou-se que existiram diferenças estatisticamente significativas na distribuição de idades, $\chi^{2}(2)=29,33 ; p<0,001$, sendo as crianças do grupo clínico-sono mais jovens.

Em relação ao sexo, no grupo de controlo a representação do sexo masculino era de $55 \%$ e no grupo de perturbação de sono $56.4 \%$. No grupo com PHDA o número de rapazes foi bastante superior ao de raparigas $(74 \%$ de rapazes). O nível educacional dos pais foi predominantemente bacharelato/licenciatura, com diferenças significativas na distribuição entre os três grupos $\chi^{2}(2)=9,711 ; p<0,001$, sendo que os pais do grupo clínico-sono apresentavam um nível educacional superior. Relativamente ao local de residência, 90,5\% das crianças pertenciam aos distritos de Lisboa e Setúbal.

A coerência interna das escalas e subescalas foi avaliada através do alfa de Cronbach (Tabela 1). Na escala completa do CSHQ-PT, o valor de alfa foi de 0,75 no grupo clínico (grupo-sono e grupo-PHDA) e 0,80 na amostra completa (grupos clínicos e grupo controlo). Relativamente à coerência interna das subescalas do CSHQ-PT, nos grupos clínicos os alfas variaram entre 0,55 e 0,85 e na amostra completa variaram entre 0,57 e 0,86 .

Como a distribuição de idades do grupo-sono e grupo-controlo foi diferente (as crianças do grupo-sono eram, em média, mais jovens), a comparação das cotações do CSHQ-PT foi realizada por classes etárias (2 - 3 anos, 4 - 5 anos e 6 - 10 anos). Relativamente ao índice de perturbação de Sono (IPS) verificaram-se valores superiores no grupo clínico-sono em todas as classes etárias. Considerando as subescalas, na classe etária 2-3 anos, encontrámos cotações superiores no grupo clínico-sono na subescala 'Duração do sono', 'Despertares noturnos', 'Parassónias' e 'Perturbação respiratória do sono'. Na classe etária dos 4 - 5 anos e 6 - 10 anos, o grupo clínico-sono apresentou

Tabela 1 - Coerência Interna (alfa de Cronbach) da Escala Completa e das Subescalas do CSHQ -PT

\begin{tabular}{lcc}
\hline Escala/Subescala & $\begin{array}{c}\text { Grupo Clínico } \\
\text { (sono + PHDA) } \\
\mathbf{n}=\mathbf{1 1 2}\end{array}$ & $\begin{array}{c}\text { Amostra Completa } \\
\text { (grupo clínico + grupo controlo) } \\
\mathbf{n}=\mathbf{1 4 8}\end{array}$ \\
\hline Escala Completa, 33 itens (IPS) & 0,75 & 0,80 \\
Subescalas: & & 0,66 \\
1. Resistência em ir para a cama & 0,64 & N.A* \\
2. Início do sono & $\mathrm{N} . A^{*}$ & 0,81 \\
3. Duração do sono & 0,81 & 0,64 \\
4. Ansiedade associada ao sono & 0,58 & 0,66 \\
5. Despertares noturnos & 0,62 & 0,57 \\
6. Parassónias & 0,55 & 0,86 \\
7. Perturbação respiratória do Sono & 0,85 & 0,65 \\
8. Sonolência diurna & 0,64 & \\
\hline
\end{tabular}

N.A*: não aplicável 


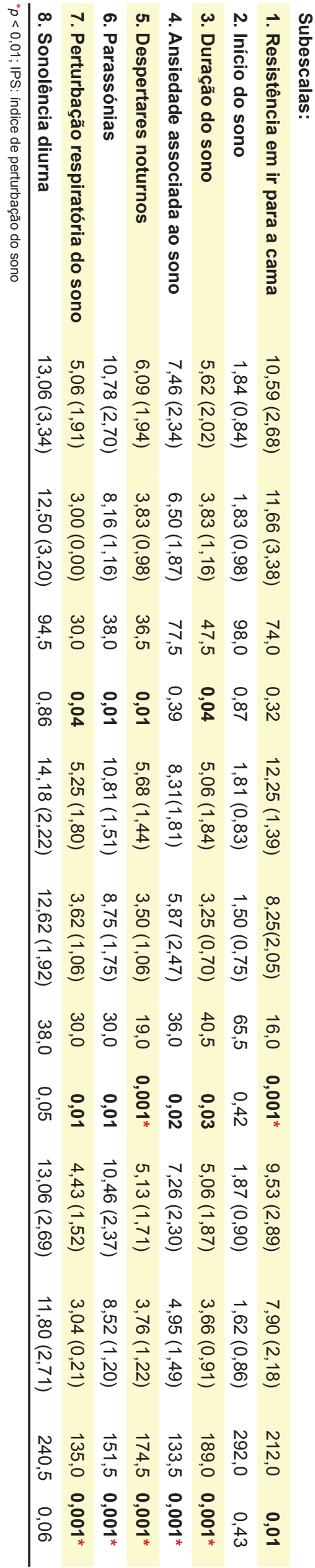

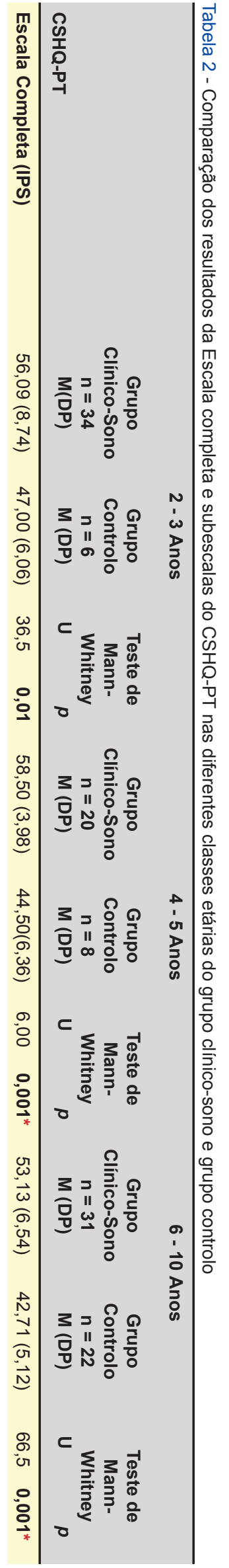

mais problemas nas subescalas 'Resistência em ir para a cama', 'Duração do sono', 'Ansiedade associada ao sono', 'Despertares noturnos', 'Parassónias' e 'Perturbação respiratória do sono'. Assim, não se encontraram diferenças significativas nas subescalas 'Início do sono' e 'Sonolência diurna' (Tabela 2).

Comparando as escalas dos três subgrupos diagnósticos de 'Perturbação do sono' (Tabela 3), verificámos que existiam diferenças significativas na cotação total e na maioria das subescalas.

Considerando os subgrupos de 'Insónia comportamental' e de 'Parassónias', encontraram-se resultados semeIhantes, com exceção da subescala 'Parassónias' (MW U $=110,0 ; p<0,01)$. Houve tendência para mais problemas com a duração do sono no subgrupo de Insónia comportamental mas não atingiu significância estatística (MW U = $225,00, p=0,15)$. Como esperado, as crianças com diagnóstico de Parassónias tiveram cotação mais elevada na respectiva subescala.

O subgrupo clínico de crianças com PROS (Tabela 3) é o que mais diferenças apresenta dos outros dois subgrupos. Como esperado, o grupo PROS apresentou uma cotação significativamente mais elevada na subescala 'Perturbação respiratória do Sono'. Apresentou cotações mais baixas (menos problemas) nas subescalas de 'Duração do sono', de 'Ansiedade associada ao sono' e de 'Despertares noturnos'.

As subescalas em que não se encontraram diferenças estatisticamente significativas entre os três subgrupos clínicos foram a de 'Resistência em ir para a cama', de 'Início do sono' e de 'Sonolência diurna'.

Comparando o grupo-PHDA com o grupo-controlo, o índice de perturbação de sono mostrou valores superiores no primeiro grupo. Considerando as subescalas, encontraram-se cotações superiores no grupo clínico-PHDA na subescala 'Ansiedade associada ao sono' e 'Parassónias' (Tabela 4).

$\mathrm{Na}$ análise receiver operating characteristic (ROC), foram consideradas as crianças com diagnóstico de perturbação do sono (grupo clínico-sono) e as crianças do grupo controlo. O valor estimado da área sob a curva foi de 0,89 (IC 95\% 0,830 - 0,949), que corresponde a um bom poder discriminativo. ${ }^{11}$ Foi determinado que um ponto de corte do IPS igual ou superior a 48 permitia identificar as crianças com distúrbio ou perturbação do sono com uma sensibilidade de 0,83 e uma especificidade de 0,69. Este ponto de corte corresponde a $30,6 \%$ das cotações mais elevadas do grupo controlo. Considerando apenas o subgrupo 4 - 10 anos, como no estudo de validação original, ${ }^{3}$ foi determinado o mesmo ponto de corte, com uma sensibilidade de 0,87 e uma especificidade de 0,76 . A aplicação deste ponto de corte ao grupo com PHDA classifica 11 casos (40,7\%) como tendo sintomas significativos de perturbação do sono.

\section{DISCUSSÃO}

O presente estudo teve como objetivo realizar a validação clínica do CSHQ-PT envolvendo 148 crianças 
Tabela 3 - Comparação dos resultados da escala completa e subescalas do CSHQ-PT entre os subgrupos do grupo clínico-sono

\begin{tabular}{|c|c|c|c|c|c|c|c|c|}
\hline \multirow[b]{2}{*}{ Escala/Subescala } & \multicolumn{2}{|c|}{$\begin{array}{c}\text { Subgrupo } 1 \\
\text { Insónia } \\
\text { Comportamental } \\
n=31\end{array}$} & \multicolumn{2}{|c|}{$\begin{array}{l}\text { Subgrupo } 2 \\
\begin{array}{l}\text { Parassónias } \\
n=19\end{array}\end{array}$} & \multicolumn{2}{|c|}{$\begin{array}{l}\text { Subgrupo } 3 \\
\begin{array}{l}\text { PROS } \\
n=35\end{array}\end{array}$} & \multirow[b]{2}{*}{ K-W $\chi^{2}$} & \multirow{2}{*}{$\begin{array}{c}\text { Comparações } \\
\text { M-W }\end{array}$} \\
\hline & M & (DP) & M & (DP) & M & (DP) & & \\
\hline Escala Completa, 33 itens (IPS) & 56,23 & $(7,04)$ & 59,11 & $(7,05)$ & 52,46 & $(6,8)$ & $9,68^{* *}$ & $\begin{array}{c}1=2 \neq 3^{* * *} \\
1 \neq 3^{*}\end{array}$ \\
\hline \multicolumn{9}{|l|}{ Subescalas: } \\
\hline 1. Resistência em ir para a cama & 10,86 & $(2,92)$ & 10,77 & $(2,88)$ & 10,03 & $(2,4)$ & $2,99 \mathrm{~ns}$ & \\
\hline 2. Inicio do sono & 1,93 & $(0,86)$ & 2,11 & $(0,83)$ & 1,60 & $(0,81)$ & $4,63 \mathrm{~ns}$ & \\
\hline 3. Duração do sono & 6,23 & $(1,94)$ & 5,44 & $(1,88)$ & 4,26 & $(1,41)$ & $17,53^{* * *}$ & $\begin{array}{c}1=2 \neq 3^{*} \\
1 \neq 3^{* *}\end{array}$ \\
\hline 4. Ansiedade associada ao sono & 8,00 & $(2,19)$ & 7,88 & $(2,19)$ & 6,93 & $(2,22)$ & $7,04^{*}$ & $\begin{array}{c}1=2 \neq 3^{*} \\
1 \neq 3^{\star *}\end{array}$ \\
\hline 5. Despertares noturnos & 6,50 & $(1,52)$ & 6,11 & $(1,90)$ & 4,50 & $(1,35)$ & $22,83^{* * *}$ & $\begin{array}{c}1=2 \neq 3 \\
1 \neq 3^{* * *}\end{array}$ \\
\hline 6. Parassónias & 10,50 & $(2,23)$ & 13,05 & $(1,89)$ & 9,40 & $(1,54)$ & $29,14^{* * *}$ & $\begin{array}{c}1 \neq 2^{* *} \neq 3^{* * *} \\
1=3\end{array}$ \\
\hline 7. Perturbação respiratória do sono & 3,80 & $(1,18)$ & 4,33 & $(1,41)$ & 6,23 & $(1,54)$ & $36,58^{* * *}$ & $\begin{array}{c}1=2 \neq 3^{* * *} \\
1 \neq 3^{* * *}\end{array}$ \\
\hline 8. Sonolência diurna & 12,80 & $(3,01)$ & 13,83 & $(3,25)$ & 13,46 & $(2,56)$ & 1,09 ns & \\
\hline
\end{tabular}

${ }^{*} p<0,05$; ${ }^{* *} p<0,01$; *** $p<0,001$; ns: não significativo

Tabela 4 - Comparação dos resultados da escala completa e subescalas do CSHQ-PT entre o grupo clínico-PHDA e o grupo controlo

\begin{tabular}{|c|c|c|c|c|c|c|}
\hline \multirow[b]{2}{*}{ Escala/Subescala } & \multicolumn{2}{|c|}{$\begin{array}{c}\text { Grupo Clínico-PHDA } \\
n=27\end{array}$} & \multicolumn{2}{|c|}{$\begin{array}{c}\text { Grupo Controlo } \\
n=36\end{array}$} & \multicolumn{2}{|c|}{$\begin{array}{c}\text { Teste de } \\
\text { Mann-Whitney }\end{array}$} \\
\hline & M & (DP) & M & (DP) & $\mathbf{U}$ & $p$ \\
\hline Escala Completa, 33 itens (IPS) & 47,70 & $(7,41)$ & 43,85 & $(5,64)$ & 334,5 & 0,04 \\
\hline \multicolumn{7}{|l|}{ Subescalas: } \\
\hline 1. Resistência em ir para a cama & 8,37 & $(2,38)$ & 8,62 & $(2,71)$ & 474,5 & 0,87 \\
\hline 2. Início do sono & 1,67 & $(0,78)$ & 1,63 & $(0,84)$ & 475,5 & 0,87 \\
\hline 3. Duração do sono & 4,03 & $(1,67)$ & 3,60 & $(0,91)$ & 459,0 & 0,65 \\
\hline 4. Ansiedade associada ao sono & 6,44 & $(1,94)$ & 5,42 & $(1,86)$ & 319,0 & 0,01 \\
\hline 5. Despertares noturnos & 4,14 & $(1,53)$ & 3,71 & $(1,12)$ & 404,0 & 0,21 \\
\hline 6. Parassónias & 9,29 & $(1,68)$ & 8,51 & $(1,31)$ & 346,0 & 0,04 \\
\hline 7. Perturbação respiratória do sono & 3,48 & $(1,01)$ & 3,17 & $(0,56)$ & 401,5 & 0,12 \\
\hline 8. Sonolência diurna & 13,2 & $(3,64)$ & 12,1 & $(2,59)$ & 396,0 & 0,20 \\
\hline
\end{tabular}

Portuguesas dos 2 aos 10 anos, divididas em três grupos: 1. Crianças com diagnóstico de perturbação do sono; 2. Crianças com diagnóstico de PHDA; 3. Grupo controlo. À semelhança do estudo de validação do questionário americano, o grupo clínico de perturbação do sono incluiu três dos diagnósticos mais frequentes: insónia comportamental da infância, parassónias e perturbação respiratória do sono.

Relativamente à coerência interna do questionário, foi determinado um valor de alfa de Cronbach da escala completa na amostra clínica (perturbações do sono e PHDA) de 0,75 , acima do valor mínimo recomendado $(0,70)$, ligeiramente abaixo da amostra clínica do estudo de validação original $(0,78)$ e da validação da versão Portuguesa em amostra comunitária $(0,78) \cdot{ }^{3,10,12}$ Considerando a amostra total, de maiores dimensões ( $n=148$ ), este valor foi um pouco superior $(0,80)$. Nas subescalas, obtivemos coerências internas na amostra clínica entre 0,55 e 0,85, semelhantes ao estudo de Owens et al, ${ }^{3}$ que variaram entre 0,44 e 0,83 . A subescala 'Parassónias' mostrou a coerência interna mais baixa $(0,55)$ à semelhança do que sucedeu noutros estudos. . $^{3,10,13,14}$ Pensamos que este facto se deve à heterogeneidade dos comportamentos que fazem parte desta escala, que não têm de surgir necessariamente associados (enurese noturna, bruxismo e pesadelos, por exemplo). Deste modo, concluímos que o CSHQ-PT apresenta propriedades psicométricas no contexto clínico que são semelhantes às que foram descritas no questionário original e adequadas para o contexto de investigação e rastreio das perturbações do sono. 
O subgrupo clínico de Insónia comportamental da infância (perturbação do sono mais frequente em idade pediátrica $)^{15}$ mostrou valores tendencialmente mais elevados nas subescalas 'Duração do sono' (insuficiente), 'Ansiedade associada ao sono' (dificuldade em adormecer de forma independente) e 'Despertares noturnos' (mais frequentes). As cotações destas subescalas foram superiores às do subgrupo com PROS. Contudo, o mesmo não se verificou relativamente ao subgrupo 'Parassónias', onde não se encontraram diferenças estatisticamente significativas. Compreendemos este resultado pela comorbilidade frequente de insónia comportamental no subgrupo 'Parassónias' (42\% na nossa amostra). De facto, é frequente que a mesma criança apresente mais de um tipo de perturbação de sono, o que dificulta a constituição de grupos homogéneos. ${ }^{4,16}$

As subescalas 'Parassónias' e 'Perturbação respiratória do sono' mostraram cotações mais elevadas nos respetivos subgrupos clínicos. A subescala 'Despertares noturnos' mostrou mais problemas a este nível nos subgrupos de 'Insónia Comportamental' e 'Parassónias' (em que ocorrem despertares parciais). Globalmente, as diferenças nas subescalas do CSHQ-PT entre os subgrupos clínicos mostram validade discriminativa na avaliação das perturbações do sono mais frequentes. Como era esperado, o IPS foi superior nos grupos clínicos quando comparado com o grupo controlo.

A análise ROC do índice de perturbação do sono permitiu determinar um ponto de corte de 48 para o rastreio das perturbações do sono, que se apresenta mais adequado para a população Portuguesa do que o proposto no estudo de validação original (41). ${ }^{3}$ Este valor foi coincidente com o recomendado por Silva et al com base na avaliação de uma amostra não clínica de crianças Portuguesas. ${ }^{4}$ Dada a predominância de itens comportamentais no questionário, a avaliação do IPS não substitui a análise das subescalas e dos itens/sintomas individuais. Para além disso, é importante salientar que o rastreio positivo não constitui um diagnóstico, que requer integração dos resultados com a anamnese, observação e eventuais exames complementares, em contexto clínico.

A comparação dos resultados do CSHQ-PT no grupo clínico-PHDA e no grupo controlo permite concluir que o IPS foi superior no grupo clínico-PHDA, assim como na subescala 'Ansiedade associada ao sono' e 'Parassónias'. A maior incidência de perturbações do sono em crianças com PHDA tem sido também descrita noutros estudos que utilizaram o CSHQ., ${ }^{57-21}$ De facto, a aplicação do ponto de corte do IPS de 48 no grupo com PHDA, corresponde uma rastreio de perturbações do sono positivo em 40,7\% dos indivíduos, valor semelhante ao que foi descrito noutro estudo $\left(44,8 \%\right.$, referente a problemas moderados do sono). ${ }^{22}$ Globalmente, é evidente que, como grupo, as crianças com PHDA apresentam mais problemas com o sono. Por este motivo, é fundamental rastrear as perturbações do sono nesta população, ainda mais porque as perturbações do sono podem desencadear sintomas semelhantes ou agra- var as características clínicas da PHDA. ${ }^{21}$

Estamos conscientes de algumas limitações neste estudo. Tal como em outros estudos de validação de questionários anteriores, foram utilizadas amostras de conveniência, que poderão não ser representativas da população em estudo. Ainda assim, um estudo prévio com crianças Portuguesas não encontrou diferenças consideráveis nos problemas do sono relacionadas com o nível educacional dos pais, com a densidade populacional da área de residência e com o local de recrutamento (escolas públicas versus hospital privado). ${ }^{6}$ Por outro lado, tal como no estudo de validação Americano, não foi garantida a avaliação clínica sistemática das perturbações do sono no grupo controlo para além do preenchimento do questionário. ${ }^{3} \mathrm{~A}$ taxa de resposta obtida $(70,9 \%)$ foi bastante razoável comparando com o estudo de validação original $(46,9 \%)$ e com o estudo de validação de Silva et al, (74\%). ${ }^{10}$ Outra limitação prende-se com a dimensão reduzida do grupo clínico- PHDA que não permite relacionar os problemas do sono com a forma de apresentação.

A validação dos questionários é um trabalho contínuo. Estudos futuros poderão caracterizar os problemas do sono associados a outras populações ou entidades clínicas e investigar a eficácia de programas de prevenção ou do tratamento das perturbações do sono, utilizando este instrumento.

\section{CONCLUSÃO}

A versão Portuguesa do Children's Sleep Habits Questionnaire mostrou propriedades psicométricas adequadas em crianças com perturbações do sono e PHDA. O ponto de corte de 48 é mais adequado para a população Portuguesa do que o que foi recomendado no estudo de validação Americano. Deste modo, apresenta-se como um instrumento útil para o rastreio e investigação das perturbações nas crianças Portuguesas.

\section{PROTECÇÃO DE PESSOAS E ANIMAIS:}

Os autores declaram que os procedimentos seguidos estavam de acordo com os regulamentos estabelecidos pelos responsáveis da Comissão de Investigação Clínica e Ética e de acordo com a Declaração de Helsínquia da Associação Médica Mundial.

\section{CONFIDENCIALIDADE DOS DADOS}

Os autores declaram ter seguido os protocolos do seu centro de trabalho acerca da publicação de dados.

\section{CONFLITOS DE INTERESSE}

Os autores declaram não ter conflitos de interesses relacionados com o presente trabalho.

\section{FONTES DE FINANCIAMENTO}

Este trabalho não recebeu qualquer tipo de suporte financeiro de nenhuma entidade no domínio público ou privado. 


\section{REFERÊNCIAS}

1. Moszczynski A, Murray JB. Neurobiological aspects of sleep physiology. Neurol Clin. 2012;30:963-85

2. Ferreira C, Ferreira H, Vieira MJ, Costeira M, Branco L, Dias Â, et al. Epidemiologia do uso de internet numa população adolescente e sua relação com hábitos de sono. Acta Med Port. 2017;30:524-33.

3. Owens JA, Spirito A, McGuinn M. The Children's Sleep Habits Questionnaire (CSHQ): psychometric properties of a survey instrument for school-aged children. Sleep. 2000;23:1043-51.

4. Silva FG, Silva CR, Braga LB, Neto AS. Hábitos e problemas do sono dos dois aos dez anos: estudo populacional. Acta Pediatr Port. 2013;44:196-202.

5. Gruber R, Fontil L, Bergmame L, Wiebe TS, Amsel R, Frenette S, et al. Contributions of circadian tendencies and behavioral problems to sleep onset problems of children with ADHD. BMC Psychiatry. 2012;12:212.

6. Silva FG. Hábitos e problemas do sono das crianças dos 2 a 10 anos Lisboa: Nova Medical School, Universidade Nova de Lisboa; 2014. p. 52-60, 106, 171-4.

7. Spruyt K, Gozal D. Pediatric sleep questionnaires as diagnostic or epidemiological tools: A review of currently available instruments. Sleep Med Rev. 2011;15:19-32

8. American Academy of Sleep Medicine. International classification of Sleep Disorders. $3^{\text {rd }}$ ed. Darien: AASM; 2014.

9. American Psychiatric Association. Diagnostic and statistical manual of mental disorders $5^{\text {th }}$ ed. Washington: APA; 2013.

10. Silva FG, Silva CR, Braga LB, Neto AS. Portuguese Children's Sleep Habits Questionnaire - validation and cross-cultural comparison. J Pediatr. 2014;90:78-84.

11. Marôco J. Análise estatística, com o SPSS Statistics. $6^{a}$ ed. Lisboa: ReportNumber; 2014.

12. Nunnally JC, Bernstein IH. Psychometric theory. $3^{\text {rd }}$ ed. New York: McGraw-Hill; 1994.

13. Tzchishinsky O, Lufi D, Shochat T. Reliability of the Children's Sleep
Habits Questionnaire Hebrew translation and cross cultural comparison of the psychometric properties. Sleep Diagnosis and Therapy. 2008;3:30-4

14. Schlarb A, Schwerdtle B, Hautzinger M. Validation and psychometric properties of the German version of the Children's Sleep Habits Questionnaire (CSHQ-DE). Somnologie. 2010;14:260-6.

15. Nunes JM, Bruni O. Insomnia in childhood and adolescence: clinical aspects, diagnosis, and therapeutic approach. J Pediatr. 2015; 91:2635.

16. Moore $\mathrm{M}$, Allison $\mathrm{D}$, Rosen $\mathrm{CL}$. A review of pediatric nonrespiratory sleep disorders. Chest. 2006;130:1252-62.

17. Abou-Khadra MK, Amin OR, Shaker OG, Rabah TM. Parent reported sleep problems, symptom ratings, and serum ferritin levels in children with attention-deficit/hyperactivity disorder: a case control study. BMC Pediatrics. 2013;13:217.

18. Yürümez E, Kılıç BG. Relationship between sleep problems and quality of life in children aith ADHD. J Atten Disord. 2016;20:34-40.

19. Vaidyanathan $S$, Shah H, Gayal T. Sleep disturbances in children with attention deficit/ hyperactivity disorder (ADHD): comparative study with healthy siblings. J Can Acad Child Adolesc Psychiatry. 2016;25:3.

20. Gomes AA, Parchão C, Almeida A, Clemente V, Pinto de Azevedo $\mathrm{MH}$. Sleep-wake patterns reported by parents in hyperactive children diagnosed according to ICD-10, as compared to paired controls. Child Psychiatry Hum Dev. 2014;45:533-43.

21. Chamorro M, Lara PJ, Inmaculada I, Espadas M, Alda-Díez, JA. Evaluación y tratamiento de los problemas de sueño en niños diagnosticados de trastorno por déficit de atención/ hiperactividad: actualización de la evidencia. Rev Neurol. 2017; 64:413-21.

22. Sung V, Hiscock H, Sciberras E, Efron D. Sleep problems in children with attention-deficit/hyperactivity disorder: prevalence and the effect on the child and family. Arch Pediatr Adolesc Med. 2008;162:336-42. 